\title{
Boundary layer flow on a long thin cylinder
}

\author{
O. R. Tutty ${ }^{\mathrm{a})}$ and W. G. Price \\ School of Engineering Sciences, University of Southampton, Southampton SO17 1BJ, United Kingdom
}

\author{
A. T. Parsons \\ QinetiQ, Winfrith Technology Centre, Dorset DT2 8XJ, United Kingdom
}

(Received 20 March 2001; accepted 18 October 2001)

\begin{abstract}
The development of the boundary layer along a long thin cylinder aligned with the flow is considered. Numerical solutions are presented and compared with previous asymptotic results. Very near the leading edge the flow is given by the Blasius solution for a flat plate. However, there is soon a significant deviation from Blasius flow, with a thinner boundary layer and higher wall shear stress. Linear normal mode stability of the flow is investigated. It is found that for Reynolds numbers less than a critical value of 1060 the flow is unconditionally stable. Also, axisymmetric modes are only the fourth least stable modes for this problem, with the first three three-dimensional modes all having a lower critical Reynolds number. Further, for Reynolds numbers above the critical value, the flow is unstable only for a finite distance, and returns to stability sufficiently far downstream. (C) 2002 American Institute of Physics. [DOI: 10.1063/1.1427921]
\end{abstract}

\section{INTRODUCTION}

In a classical analysis of the flow in an external boundary layer the pressure gradient is obtained from the inviscid (potential) solution for flow past the body. The simplest example is Blasius flow past a flat plate aligned with the flow in which the pressure gradient is zero. Consider the case of a circular cylinder with the external flow aligned along the cylinders axis. If the cylinder is solid, then the flow will adjust to the presence of the cylinder, generating a nonzero pressure gradient near the nose of the cylinder. However the pressure gradient will decay asymptotically along the cylinder. Alternatively, if fluid is being sucked into the cylinder at the free stream velocity, the pressure gradient will zero from the leading edge.

Apart from its intrinsic interest, the flow along a long cylinder could be regarded as an idealized model of the flow along a thread. However, our interest arose from a different problem, that of flow along towed sonar arrays which are used for underwater sensing. These devices are cylindrical in shape and can have nondimensional lengths of $O\left(10^{5}\right)$ or greater.

The problem of flow developing along a circular cylinder with zero pressure gradient will be considered here. There is little reported in the literature on this problem. Seban and Bond ${ }^{1}$ give the first three terms in a series solution valid near the leading edge of the cylinder, giving in particular expressions for the shear stress on the surface and the displacement area. Kelly ${ }^{2}$ presents different values for some of the coefficients in the Seban and Bond solution. In contrast, Stewartson ${ }^{3}$ gives a series solution for very large distances along the cylinder. In Ref. 3 it is shown that sufficiently far along the cylinder, the wall shear stress decays logarithmically with distance, rather than algebraically as is

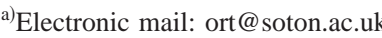

usually found. Glauert and Lighthill ${ }^{4}$ considered the flow along the entire cylinder. They developed a series solution similar to that of Stewartson for the flow far downstream. Also, they developed an approximate solution for flow near the leading edge based on a Pohlhausen method with a logarithmic profile, and have shown that this solution produces reasonable agreement with different series solutions valid near the leading edge and far downstream. Using these solutions they produced a set of recommended curves for quantities such as the displacement area and the skin friction.

Here a full numerical solution of the boundary layer problem will be presented and compared with previous results. In addition, the stability of the flow to linear normal modes will be considered. Since three-dimensional disturbances in cylindrical coordinates cannot be reduced to an equivalent axisymmetric disturbance by a transformation of Squire's type, it is necessary to consider three-dimensional as well as axisymmetric modes.

\section{FORMULATION}

Assuming zero pressure gradient, the boundary layer equations in polar coordinates $(x, r)$, nondimensionalized on the cylinder radius, are

$$
\begin{aligned}
& \frac{\partial u}{\partial x}+\frac{\partial v}{\partial r}+\frac{v}{r}=0, \\
& u \frac{\partial u}{\partial x}+v \frac{\partial u}{\partial r}=\frac{1}{\operatorname{Re}}\left(\frac{\partial^{2} u}{\partial r^{2}}+\frac{1}{r} \frac{\partial u}{\partial r}\right),
\end{aligned}
$$

where $(u, v)$ are the stream wise and radial velocity components, nondimensionalized on the free stream velocity $U_{\infty}$. $\operatorname{Re}=U_{\infty} a / \nu$ is the Reynolds number where $\nu$ is the kinematic viscosity.

Introducing the transformation

$$
y=(r-1) \operatorname{Re}^{1 / 2}, \quad v \rightarrow \operatorname{Re}^{-1 / 2} v,
$$


which incorporates the usual $\mathrm{Re}^{-1 / 2}$ scaling, produces

$$
\begin{aligned}
& \frac{\partial u}{\partial x}+\frac{\partial v}{\partial y}+\frac{v \operatorname{Re}^{-1 / 2}}{1+\operatorname{Re}^{-1 / 2} y}=0, \\
& u \frac{\partial u}{\partial x}+v \frac{\partial u}{\partial y}=\frac{\partial^{2} u}{\partial y^{2}}+\frac{\operatorname{Re}^{-1 / 2}}{1+\operatorname{Re}^{-1 / 2} y} \frac{\partial u}{\partial y},
\end{aligned}
$$

with boundary conditions

$$
\begin{aligned}
& u=v=0 \quad \text { at } y=0, \\
& u \rightarrow 1 \quad \text { as } y \rightarrow \infty .
\end{aligned}
$$

In addition a suitable initial condition must be specified. This will be considered below.

\section{THEORETICAL RESULTS}

From (4)-(7) it may appear that the leading term in the boundary layer solution will be flat plate Blasius flow, with an $O\left(\mathrm{Re}^{-1 / 2}\right)$ perturbation. While this is true near the leading edge of the cylinder, further downstream this approximation beaks down. With the Blasius solution, $y$ scales as $x^{1 / 2}$. Hence the ratio of the terms on the right-hand side of (5) is

$$
\frac{\operatorname{Re}^{-1 / 2}}{1+\operatorname{Re}^{-1 / 2} y} \frac{\partial u}{\partial y} / \frac{\partial^{2} u}{\partial y^{2}}=O\left(\frac{\operatorname{Re}^{-1 / 2} x^{1 / 2}}{1+\operatorname{Re}^{-1 / 2} x^{1 / 2}}\right) .
$$

Hence when $x=O(\mathrm{Re})$ the terms of the right-hand side are of similar magnitude due to the growth in the boundary layer, and Blasius flow will not be the leading term in the solution when the boundary layer thickness becomes comparable with the cylinder radius. Further, at this stage it is also necessary to include the additional term in the continuity equation at leading order.

Here the extra term will be included from the leading edge by adopting the boundary layer equations in the form (4)-(7). These equations will be solved numerically, using the method outlined below. First, however, the first two terms in the series solution for the flow near the leading edge, valid in the region $0<x \ll \mathrm{Re}$, will be presented. Following Seban and Bond, ${ }^{1}$ take

$$
\eta=\operatorname{Re}^{1 / 2} x^{-1 / 2}\left(r^{2}-1\right) / 2, \quad \xi=\operatorname{Re}^{-1 / 2} x^{1 / 2},
$$

and

$$
\psi=\operatorname{Re}^{-1 / 2} x^{1 / 2} f(\xi, \eta) .
$$

This gives

$$
\begin{aligned}
& u=\frac{1}{r} \frac{\partial \psi}{\partial r}=\frac{\partial f}{\partial \eta}, \\
& v=-\frac{1}{r} \frac{\partial \psi}{\partial x}=-\frac{\operatorname{Re}^{-1 / 2} x^{-1 / 2}}{r}\left[-\eta \frac{\partial f}{\partial \eta}+\frac{\partial}{\partial \xi}(\xi f)\right] .
\end{aligned}
$$

The governing equation for $f$ is

$$
\begin{aligned}
& \frac{\partial}{\partial \eta}\left[(1+2 \xi \eta) \frac{\partial^{2} f}{\partial \eta^{2}}\right]+\frac{1}{2} f \frac{\partial^{2} f}{\partial \eta^{2}}+\frac{1}{2} \xi\left(\frac{\partial f}{\partial \xi} \frac{\partial^{2} f}{\partial \eta^{2}}\right. \\
& \left.+\frac{\partial f}{\partial \eta} \frac{\partial^{2} f}{\partial \eta \partial \xi}\right)=0 .
\end{aligned}
$$

For $\xi \ll 1$ take

$$
f=f_{0}(\eta)+\xi f_{1}(\eta)+\cdots
$$

which produces

$$
\begin{aligned}
& f_{0}^{\prime \prime \prime}+\frac{1}{2} f_{0} f_{0}^{\prime \prime}=0, \\
& f_{1}^{\prime \prime \prime}+\frac{1}{2} f_{0} f_{1}^{\prime \prime}-\frac{1}{2} f_{0}^{\prime} f_{1}^{\prime}+f_{0}^{\prime \prime} f_{1}+2 \eta f_{0}^{\prime \prime \prime}+2 f_{0}^{\prime \prime}=0 .
\end{aligned}
$$

The boundary conditions are

$$
\begin{aligned}
& f_{0}(0)=f_{0}^{\prime}(0)=0 \quad \text { and } \quad f_{0}^{\prime} \rightarrow 1 \quad \text { as } \eta \rightarrow \infty, \\
& f_{1}(0)=f_{1}^{\prime}(0)=0 \quad \text { and } \quad f_{1}^{\prime} \rightarrow 0 \quad \text { as } \quad \eta \rightarrow \infty .
\end{aligned}
$$

Equation (14) is the Blasius equation. The problems defined by (14)-(17) are easily solved numerically. The solution gives the dimensionless skin friction $\tau=(\partial u / \partial y)_{y=0}$ as

$$
\tau=0.332 x^{-1 / 2}+0.694 \mathrm{Re}^{-1 / 2}+\cdots .
$$

This is essentially the same expression given by Seban and Bond/Kelly, ${ }^{1,2}$ but with a small difference in the second coefficient, due presumably to the increased accuracy of the calculations performed here. Equation (18) has the skin friction tending to a constant for large $x$. It is however valid only for $x \ll \operatorname{Re}$. In fact, by the point $x / \operatorname{Re}=1 / 4$ the second term in (18) is the larger.

The asymptotic series produced by Glauert and Lighthill $^{4}$ for large $x$ gives

$$
\tau=\frac{2}{\delta}+\frac{2 \gamma}{\delta^{2}}+\frac{2 \gamma^{2}-\frac{1}{2} \pi^{2}-4 \ln 2}{\delta^{3}}+O\left(\delta^{-4}\right),
$$

where $\gamma=0.5772$ is Euler's constant and $\delta=\ln (4 x / \operatorname{Re})$. This formula has $\tau$ decaying as $x$ increases, albeit slowly, inversely with $\ln x$. Stewartson ${ }^{3}$ produced a similar expression, but used $\hat{\delta}=\ln (4 x / \operatorname{Re} C)$ in place of $\delta$, where $\ln C=\gamma$. Stewartson's formula is

$$
\tau=\frac{2}{\hat{\delta}}-\frac{\frac{1}{2} \pi^{2}+4 \ln 2}{\hat{\delta}^{3}}+\cdots+\frac{\operatorname{Re}}{2 x} \frac{7}{\hat{\delta}}+\cdots .
$$

Equation (19) can be derived from Stewartson's expression by writing $\hat{\delta}=\delta-\gamma$ and expanding the first two terms in (20) for large $x$. The Glauert and Lighthill expression for $\tau$ is used here as it gives a better comparison with the numerical solutions presented below than Stewartson's formula.

Another of quantity of interest is the "displacement area," which represents the amount by which the fluid in the main stream is displaced by the action the viscous effects in the boundary layer. In two-dimensional flow the displacement thickness gives the distance which the streamlines in the far field are displaced from those of the inviscid flow past the body. However, in cylindrical coordinates, the displacement of the streamlines away from the surface decays inversely with $r$ due to the expansion in area with $r$. Hence there is no single displacement thickness, while there is a specific displacement area. In nondimensional form

$$
\Delta_{1}=2 \int_{1}^{\infty}(1-u) r d r
$$


gives the displacement area relative to the cross-sectional area of the cylinder. The displacement of the streamlines in the far field is given by

$$
\delta_{1}=r\left[1+\frac{\Delta}{r^{2}}\right]^{1 / 2}-r=\frac{\Delta}{2 r}-\frac{\Delta^{2}}{8 r^{3}}+\cdots,
$$

where the inviscid streamline is at $r$ and the equivalent viscous streamline at $r+\delta_{1}$.

Substituting the Blasius profile directly into (21) produces

$$
\Delta_{1}=\operatorname{Re}^{-1 / 2} x^{1 / 2}\left[3.442+4.37 \operatorname{Re}^{-1 / 2} x^{1 / 2}\right] .
$$

The solution to (14)-(17) gives

$$
\Delta_{1}=\operatorname{Re}^{-1 / 2} x^{1 / 2}\left[3.442+0.143 \operatorname{Re}^{-1 / 2} x^{1 / 2}+\cdots\right] .
$$

Equation (24) is valid only for $0<x \ll \operatorname{Re}$. For large $x$ Glauert and Lighthill give

$$
\Delta_{1}=\frac{4 x}{\operatorname{Re}}\left[\frac{1}{\delta}+\frac{1+\gamma+2 \ln 2}{\delta^{2}}+\cdots\right] .
$$

Stewartson $^{3}$ states that the boundary layer thickness is ultimately of order $(x / \operatorname{Re} \hat{\delta})^{1 / 2}$, consistent with (25). Hence the boundary layer grows at a factor $(\ln x)^{-1 / 2}$ more slowly than for the flat plate.

\section{NUMERICAL METHOD}

In the original polar coordinates the velocity can be obtained from the stream function $\psi(x, r)$ through

$$
u=\frac{1}{r} \frac{\partial \psi}{\partial r}, \quad v=-\frac{1}{r} \frac{\partial \psi}{\partial x} .
$$

In boundary layer coordinates write

$$
\psi=\operatorname{Re}^{-1 / 2} \Psi(x, z),
$$

where $z=\operatorname{Re}^{1 / 2} x^{-1 / 2}(r-1)$. The boundary layer equations (4) and (5) can now be written as a system of coupled first order differential equations:

$u=\frac{x^{-1 / 2}}{1+\operatorname{Re}^{-1 / 2} x^{1 / 2} z} \frac{\partial \Psi}{\partial z}$,

$\tau=x^{-1 / 2} \frac{\partial u}{\partial z}$

$$
\begin{aligned}
u \frac{\partial u}{\partial x} & -\frac{1}{1+\operatorname{Re}^{-1 / 2} x^{1 / 2} z} \frac{\partial \Psi}{\partial x} \tau \\
& =x^{-1 / 2} \frac{\partial \tau}{\partial z}+\frac{\operatorname{Re}^{-1 / 2}}{1+\operatorname{Re}^{-1 / 2} x^{1 / 2} z} \tau .
\end{aligned}
$$

The boundary conditions are

$$
\Psi(x, 0)=u(x, 0)=0 \quad \text { and } \quad u \rightarrow 1 \quad \text { as } \eta \rightarrow \infty .
$$

The Keller box ${ }^{5}$ method is used to solve (28)-(31). The Keller box method is a Crank-Nicolson finite difference method, which is second order accurate. Newton's method is used to solve the nonlinear set of algebraic equations which result once the equations have been discretized. A uniform

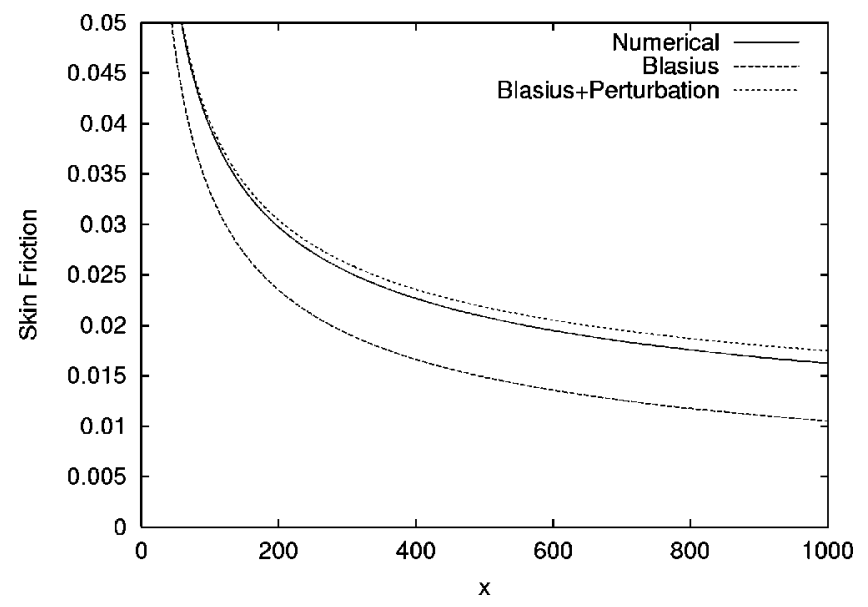

FIG. 1. Dimensionless skin friction near the leading edge of the cylinder for $\operatorname{Re}=10^{4}$

grid is used in $z$. Since the Keller box method involves values only at the present and previous grid points in the streamwise direction, the grid step in $x$ can be changed with no further complications to the method. The streamwise grid step is scaled with $x^{1 / 2}$ in accordance with the expected development of the boundary layer, so that $\Delta x=x^{1 / 2} \Delta$.

The initial condition was obtained from calculating $u$ from (10), (13), and the solution to (14)-(17), and then using (28) and (30) to estimate $\Psi$ and $\tau$. Typically the far field conditions were enforced at $z_{\max }=30$ with 3000 points in $z$, $\Delta=0.01$, and $x_{0}=0.01$. Note that the large number of points in $z$ were required by the stability calculation, not that for the flow field where an order of magnitude fewer points could have been used.

\section{RESULTS}

For reference consider a flow with $\mathrm{Re}=10^{4}$, corresponding, e.g., to a cylinder with diameter $1 \mathrm{~cm}$ in water with a free stream velocity of $1 \mathrm{~m} / \mathrm{s}$. Figure 1 shows the dimensionless skin friction near the leading edge of the cylinder where the Blasius solution should be valid at leading order. Figure 1 shows $\tau(x, 0)$ from the numerical solution, the Blasius values and Blasius plus the $O\left(\mathrm{Re}^{-1 / 2}\right)$ perturbation. Clearly, even at this stage the perturbation is significant, and the Blasius solution alone does not give an accurate estimate of the skin friction.

Figure 2 shows the skin friction much further downstream. For very large $x$, the Blasius values are much too small while the addition of the $O\left(\mathrm{Re}^{-1 / 2}\right)$ term gives values that are too large. For sufficiently large $x$ the Glauert and Lighthill formula (19) gives a good estimate of the skin friction.

The displacement area is shown in Fig. 3. This figure displays $\Delta_{1}$ calculated directly from the numerical solution, using the Blasius solution (23), the expansion valid for 0 $<x \ll \operatorname{Re}(24)$, and Glauert and Lighthill's expression for large $x$ (25). As expected $\Delta_{1}$ is smaller than that given using the Blasius profile, and (24) is valid only near the leading edge of the cylinder. Glauert and Lighthill's formula (25) is tending slowly towards the numerical values for very large $x$. 


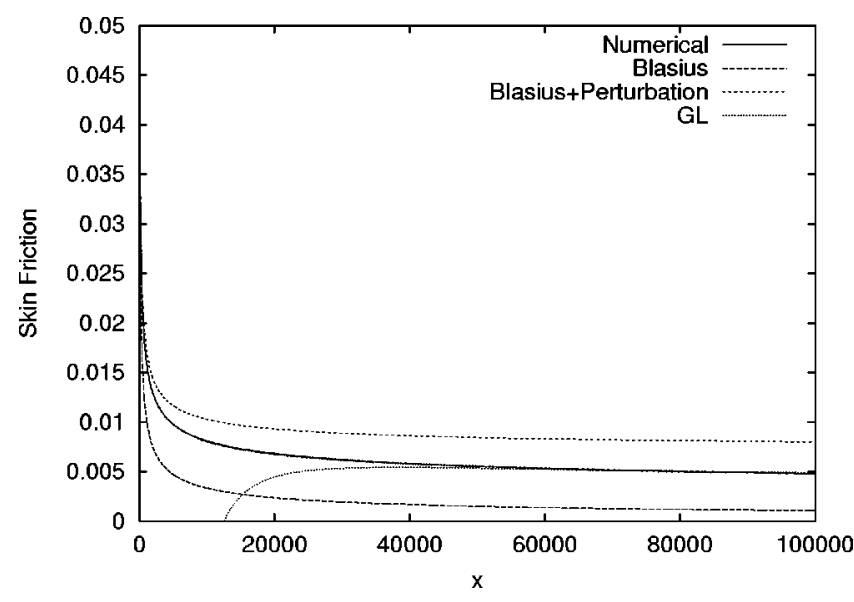

FIG. 2. Dimensionless skin friction for $\mathrm{Re}=10^{4}$.

Perhaps of more interest than the displacement area, which does not give a direct measure of the displacement of the streamlines, is the boundary layer thickness. This is shown in Fig. 4, along with the Blasius values. The boundary layer thickness has been defined as the value of $y=x^{1 / 2} z$ where $u / U_{\infty}=0.99$. The boundary layer is significantly thinner than for the flat plate case, with the difference increasing with $x$. This is consistent with the higher shear stress in the cylindrical case.

The streamwise velocity profiles with $\mathrm{Re}=10^{4}$ for $x$ $=0.01$ to $x=10^{5}$ in evenly spaced intervals of $x^{1 / 2}$ are shown in Fig. 5. The velocity is plotted against $z$ so the figure shows the change in shape of the velocity profile as the radial effects become more important. This can also be seen in Fig. 6 which shows the scaled shear stress $\partial u / \partial z$ versus $z$. Near the leading edge, where the flow is close to Blasius flow, the shear near the surface is approximately constant, but further downstream, the velocity near the surface changes nonlinearly. In fact, this can be deduced from (30) and which gives $\partial \tau / \partial z=-x^{1 / 2} \operatorname{Re}^{-1 / 2} \tau$ at $z=0$. Far downstream, with (19), this gives

$$
\frac{\partial \tau}{\partial z}=-\frac{(4 x / \mathrm{Re})^{1 / 2}}{\ln (4 x / \mathrm{Re})}+\cdots \quad \text { at } z=0
$$

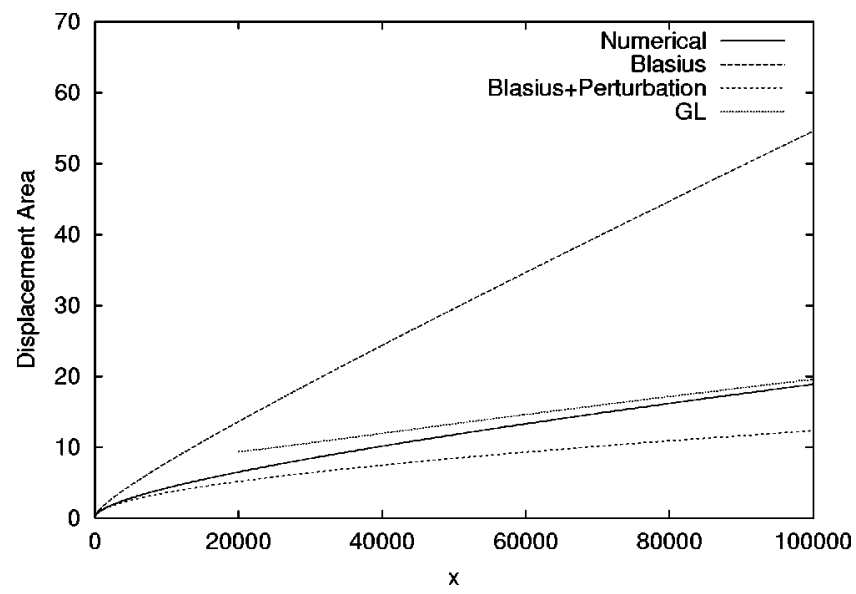

FIG. 3. Displacement area for $\mathrm{Re}=10^{4}$.

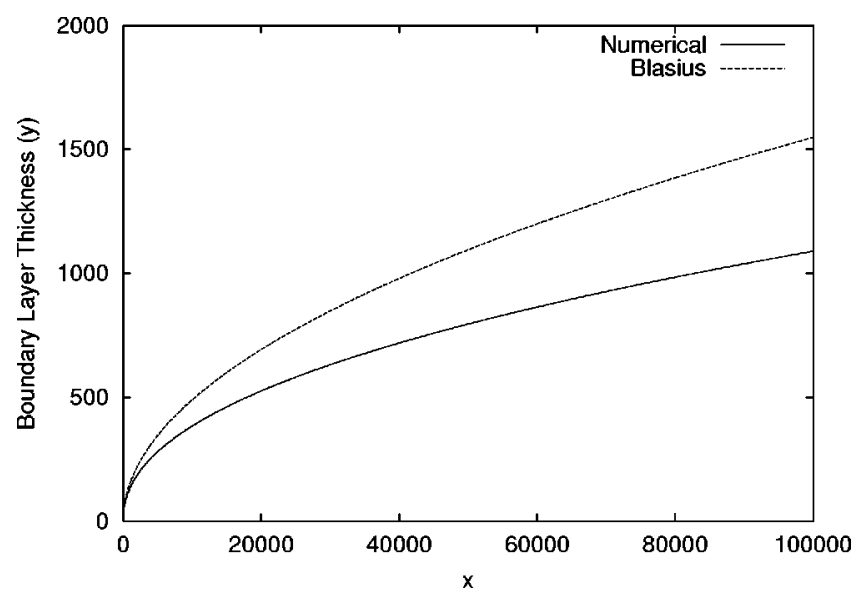

FIG. 4. Dimensionless boundary layer thickness for $\mathrm{Re}=10^{4}$.

and hence since the magnitude of $\partial \tau / \partial z$ increases continuously with $x$, the change in the velocity near the surface becomes more and more nonlinear as $x$ increases, consistent with Fig. 6.

The radial velocity scaled by $x^{1 / 2} \mathrm{Re}^{1 / 2} v$, is plotted against $z$ in Fig. 7. At $x=0.01, v$ increases to a maximum near $z=5$ with a slight decrease for greater $z$ as far as $z_{\max }$ $=20$. Further downstream there is a peak in $v$ near the wall with a more noticeable decay further away from the wall. In fact, this decay is to be expected; from the continuity equation (1), it is clear that $v$ must eventually decay inversely proportionally to $r$. This will occur at any value of $x$ if $r$ is sufficiently large. The apparent near constant value of $v$ for large $z$ seen in Fig. 6 for $x=0.01$ is consistent with the flow being close to Blasius flow and $r_{\max }-1$ being small (0.02) at this point.

\section{STABILITY: AXISYMMETRIC MODES}

Flat plate boundary layer flow is one of the cases in which linear stability theory based on a normal mode approach assuming locally parallel flow produces a reasonable

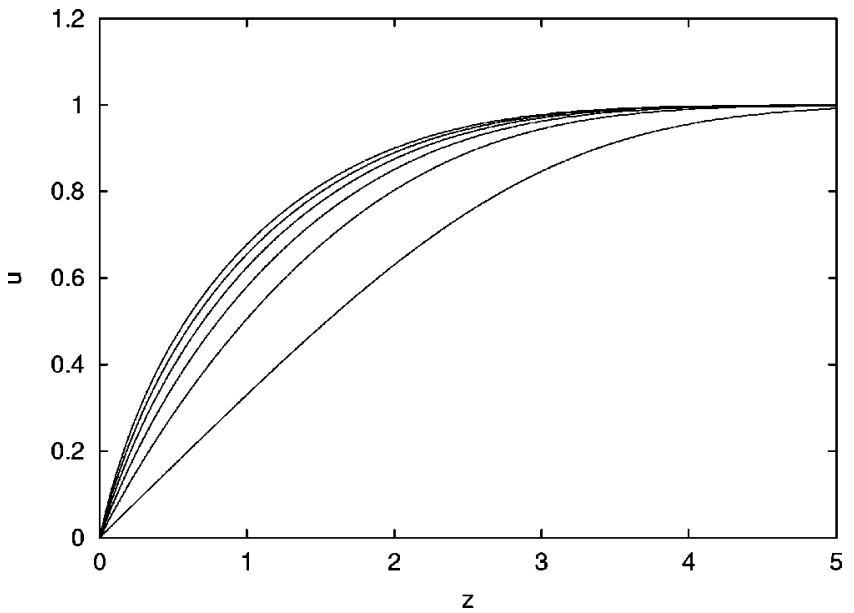

FIG. 5. Streamwise velocity $u$ for $x=0.01$ (lowest curve) to $x=10^{5}$ (highest curve) plotted against $z \cdot \operatorname{Re}=10^{4}$. 


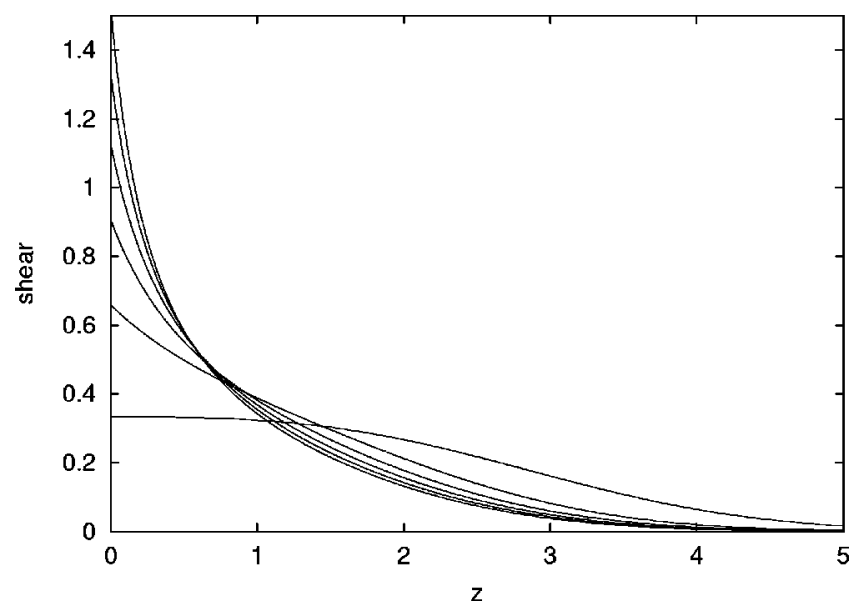

FIG. 6. Scaled shear stress $\partial u / \partial z$ for $x=0.01$ (lowest value at $z=0$ ) to $x$ $=10^{5}$ (highest at $z=0$ ) plotted against $z \cdot \operatorname{Re}=10^{4}$.

comparison with experimental results. Hence, for very high values of Re where the flow near the leading edge of the cylinder is given by Blasius flow, linear theory should indicate where the flow first becomes unstable in this case as well. For lower Re or further down the cylinder, the higher values of the wall shear stress and lower values of the boundary layer thickness suggest that the flow will be more stable for the cylinder than the flat plate. However, the governing equation for the disturbance is not the same, so this prediction must be treated with caution.

The disturbance to the flow is given by

$$
\psi_{1}=\phi(r) \exp [i(\alpha x-\omega t)]
$$

where $\psi_{1}$ is the disturbance stream function, $\phi(r)$ its complex amplitude, and $\alpha$ and $\omega$ the wave number and frequency of the disturbance. Substituting (33) in the NavierStokes equations and linearizing produces

$$
(u-c)\left(D-\alpha^{2}\right) \phi-r\left(\frac{u^{\prime}}{r}\right)^{\prime} \phi=\frac{1}{i \alpha \operatorname{Re}}\left(D-\alpha^{2}\right)^{2} \phi,
$$

where

$$
D \equiv \frac{\partial^{2}}{\partial r^{2}}-\frac{1}{r} \frac{\partial}{\partial r}
$$

and $c=\omega / \alpha=c_{r}+i c_{i}$ is the complex wave speed. Equation (34) is the axisymmetric equivalent of the more familiar Orr-Sommerfeld equation found for two-dimensional flow.

The boundary conditions for $\phi$ are the usual zero velocity condition at the wall ( $\phi=\partial \phi / \partial r=0$ at $r=1)$ and for the disturbance to vanish in the far field.

Equation (34) was solved using a standard second order finite difference method with a uniform grid. A Newton method was used to solve the discrete equations at each iteration. The only problem that arises in using this method is in specifying a form for the outer boundary conditions. Consider (34) in the far field where $u$ is asymptotically close to one. Taking $\phi=r \hat{\phi}$ it can be shown that the decaying solu-

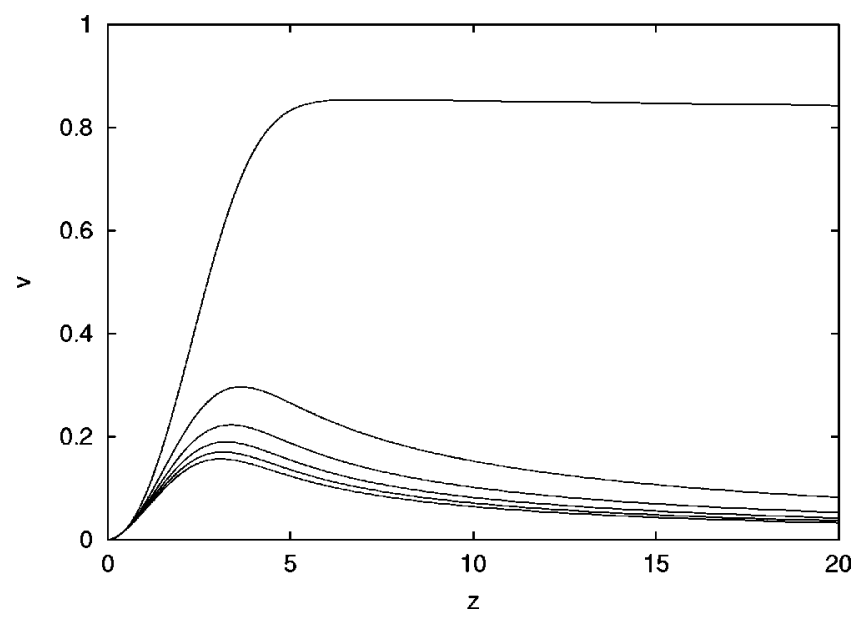

FIG. 7. Radial velocity $x^{1 / 2} \operatorname{Re}^{1 / 2} v$ for $x=0.01$ (top curve) to $x=10^{5}$ (bottom curve) plotted against $z$. Re $=10^{4}$.

tions for $\hat{\phi}$ take the form of $K_{1}(\alpha r)$ and $K_{1}(\hat{\alpha} y)$, where $K_{1}$ is the modified Bessel function of order one and $\hat{\alpha}=\left[\alpha^{2}\right.$ $+i \alpha(u-c) \mathrm{Re}]^{1 / 2}$. For large argument, $K_{1}(z) \sim z^{-1 / 2}$ $\times \exp (-z)$. For the modes of interest $\mathfrak{R}(\hat{\alpha})>\alpha$, and hence the slowest decaying disturbance in the far field has the form $\phi \sim r^{1 / 2} \exp (-\alpha r)$. This behavior can be used to derive Neumann conditions which can be applied at $r=r_{\max }$. In particular, assuming that $\alpha r$ is large, we can use

$$
\frac{\partial \phi}{\partial r}=-\alpha \phi
$$

A nontrivial solution was forced by setting $\phi$ to a (arbitrary) small value in the far field. The position of the far field boundary was varied to ensure that the results were not affected by the value used for $r_{\max }$.

The code used to obtain $\phi$ and $c$ was adapted from a standard Orr-Sommerfeld solver in Cartesian coordinates which has been thoroughly checked against results from the literature, such as those for Blasius flow, plane Poiseuille flow, and a flat plate boundary layer with surface suction. In addition, the results obtained using a perturbation stream function agree with those using a velocity-pressure formulation (details are given in the next section). A very large number of points (up to $64 \times 10^{3}$ ) was used to check the accuracy of the results, particularly for the critical Reynolds numbers, which are believed to be accurate to the figures quoted. Note that it would have been possible to use less points by changing the numerical method, e.g., by stretching the grid or using a spectral method, but since the calculations can be performed on a standard desktop machine there was no point in adopting a more complicated/sophisticated method.

Adopting the boundary layer scaling

$$
r=1+\operatorname{Re}^{-1 / 2} y, \quad \alpha=\operatorname{Re}^{1 / 2} \beta,
$$

Eq. (34) becomes 


$$
\begin{aligned}
(u-c) & \left(\frac{\partial^{2} \phi}{\partial y^{2}}-\frac{\operatorname{Re}^{-1 / 2}}{r} \frac{\partial \phi}{\partial y}-\beta^{2} \phi\right)-u^{\prime \prime} \phi+\frac{\operatorname{Re}^{-1 / 2}}{r} u^{\prime} \phi \\
= & \frac{1}{i R_{l} \beta}\left[\frac{\partial^{4} \phi}{\partial y^{4}}-\frac{2 \mathrm{Re}^{-1 / 2}}{r} \frac{\partial^{3} \phi}{\partial y^{3}}+\frac{3 \operatorname{Re}^{-1}}{r^{2}} \frac{\partial^{2} \phi}{\partial y^{2}}\right. \\
& \left.-\frac{3 \operatorname{Re}^{-3 / 2}}{r^{3}} \frac{\partial \phi}{\partial y}-2 \beta^{2}\left(\frac{\partial^{2} \phi}{\partial y^{2}}-\frac{\operatorname{Re}^{-1 / 2}}{r} \frac{\partial \phi}{\partial y}\right)+\beta^{4} \phi\right],
\end{aligned}
$$

where $R_{l}=\mathrm{Re}^{1 / 2}$, and the prime on $u$ in (38) refers to $\partial / \partial y$.

From (38) it is clear that for large Re the stability characteristics near the leading edge of the cylinder should be similar to those for the flat plate. Drazin and Reid ${ }^{6}$ give the critical values of

$$
R_{p}=519, \quad \alpha_{p}=0.304, \quad c_{r_{p}}=0.3966
$$

based on the displacement thickness as the characteristic length. In nondimensional terms the displacement thickness for Blasius flow is given by

$$
\delta_{1}=1.72 x^{1 / 2} \operatorname{Re}^{-1 / 2} .
$$

Hence in the scalings used here

$$
x_{c}=\left(\frac{R_{p}}{1.72}\right)^{2} / \operatorname{Re} \text { and } \alpha_{c}=\frac{\alpha_{p} \operatorname{Re}^{1 / 2}}{1.72 x_{c}^{1 / 2}},
$$

where $x_{c}$ is the point the flow first becomes unstable.

Calculations were performed for a range of Reynolds numbers. For $\mathrm{Re}=10^{5}$, when (41) gives $x_{c}=0.91$ and $\alpha_{c}$ $=58.5$, the critical point was found to be at $x_{c} \approx 0.99$ with $\alpha_{c} \approx 56.0$. As expected, this is further downstream than for Blasius flow. For $\operatorname{Re}=5 \times 10^{4},(41)$ gives $x_{c}=1.82$ and $\alpha_{c}$ $=29.3$, while for the cylinder, $x_{c} \approx 2.18$ with $\alpha_{c} \approx 26.7$.

The gap between the predicted and calculated values of $x_{c}$ increases as Re decreases. For $\mathrm{Re}=2 \times 10^{4}$ (41) gives $x_{c}$ $=4.55$ and $\alpha_{c}=11.7$, while the values from the numerical solution are $x_{c} \approx 8.02$ and $\alpha_{c} \approx 8.73$. However, for $\operatorname{Re}$ $<12439$ no unstable modes were found. Note that at this point the eigenvalue $c$ is consistent with the values for higher Re, which indicates that the solution to (38) has not jumped to another branch, i.e., is still obtaining the least stable solution. At the critical point with $\mathrm{Re}=12439, c_{r}=0.317$ and $\alpha=2.73$.

Figure 8 shows $c_{i}$ against $x$ for Reynolds numbers at and near the critical Reynolds number. Clearly for these Reynolds numbers there is a point along the cylinder at which the flow is maximally unstable, with the flow becoming monotonically more stable further downstream. This pattern was found for all Reynolds numbers investigated, with the flow unstable only for a finite section of the cylinder if at all. That is, if the flow is unstable then $c_{i}>0$ only for $x_{c}<x$ $<x_{s}$, where $x_{s}$ depends on $\mathrm{Re}$. For $\mathrm{Re}=2 \times 10^{4}, x_{s} \approx 4.2$ $\times 10^{2}$, for $\operatorname{Re}=5 \times 10^{4}, x_{s} \approx 4.1 \times 10^{3}$, and for $\mathrm{Re}=10^{5}, x_{s}$ $\approx 2.2 \times 10^{4}$. In all cases investigated the change back to stable flow is still in the region in which the series with Blasius flow as the leading term might be expected to be valid.

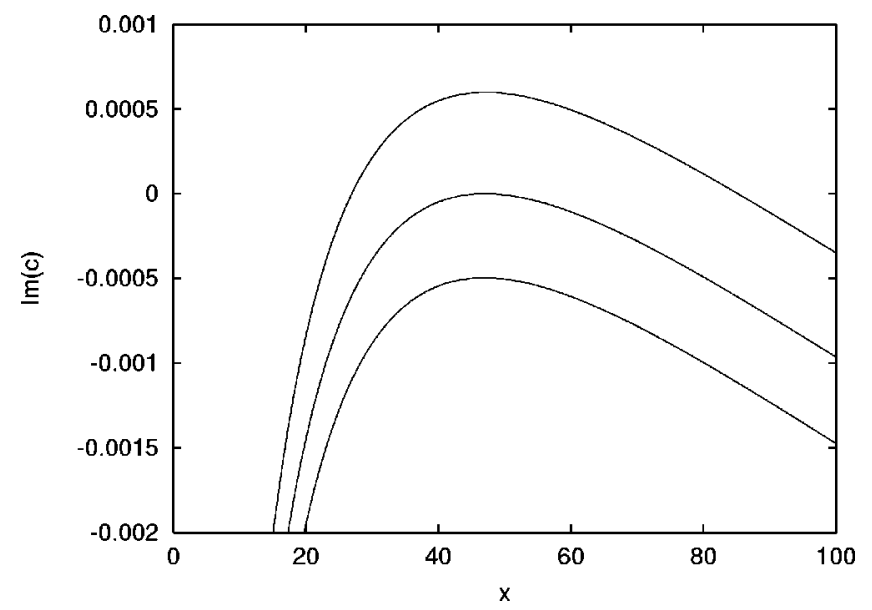

FIG. 8. The maximum value of $c_{i}$ for axisymmetric modes versus $x$. Re $=12000$ (bottom), $\operatorname{Re}=12439$ (middle), and $\mathrm{Re}=13000$ (top).

\section{STABILITY: THREE-DIMENSIONAL MODES}

For parallel flow, normal mode stability in Cartesian coordinates it is usual to consider only two-dimensional disturbances as Squires theorem ${ }^{6}$ shows that the minimum critical Reynolds number arises with two-dimensional modes. However, in cylindrical polar coordinates, three-dimensional disturbances cannot be reduced by a transformation of Squire's type to an equivalent axisymmetric disturbance, so that Squire's theorem cannot be invoked. Hence it is necessary to consider full three-dimensional disturbances.

Let the disturbance have the form

$$
(\hat{u}, \hat{v}, \hat{w}, \hat{p})(r) \exp [i(\alpha x+n \theta-\omega t)]
$$

where $(\hat{u}, \hat{v}, \hat{w})$ give the velocity components in polar coordinates $(x, r, \theta), \hat{p}$ the pressure perturbation, and $n$ is an integer. Then, assuming parallel flow, the governing equations are

$$
\begin{aligned}
& i \alpha \hat{u}+\frac{1}{r} \frac{\partial}{\partial r}(r \hat{v})+\frac{i n}{r} \hat{w}=0 \\
& i \alpha(u-c) \hat{u}+\frac{\partial u}{\partial r} \hat{v}=-i \alpha \hat{p}+\frac{1}{\operatorname{Re}}\left[\frac{\partial^{2} \hat{u}}{\partial r^{2}}+\frac{1}{r} \frac{\partial \hat{u}}{\partial r}\right. \\
& \left.-\left(\alpha^{2}+\frac{n^{2}}{r^{2}}\right) \hat{u}\right] \\
& \begin{aligned}
i \alpha(u-c) \hat{v}= & -\frac{\partial \hat{p}}{\partial r}+\frac{1}{\operatorname{Re}}\left[\frac{\partial^{2} \hat{v}}{\partial r^{2}}+\frac{1}{r} \frac{\partial \hat{v}}{\partial r}-\left(\alpha^{2}+\frac{n^{2}}{r^{2}}\right) \hat{v}\right. \\
& \left.-\frac{1}{r^{2}} \hat{v}-\frac{2 i n}{r^{2}} \hat{w}\right], \\
i \alpha(u-c) \hat{w}= & -\frac{i n}{r} \hat{p}+\frac{1}{\operatorname{Re}}\left[\frac{\partial^{2} \hat{w}}{\partial r^{2}}+\frac{1}{r} \frac{\partial \hat{w}}{\partial r}-\left(\alpha^{2}+\frac{n^{2}}{r^{2}}\right) \hat{w}\right. \\
& \left.+\frac{2 i n}{r^{2}} \hat{v}-\frac{1}{r^{2}} \hat{w}\right]
\end{aligned}
\end{aligned}
$$


where again $c=\omega / \alpha$.

The boundary conditions are again zero velocity at the surface and the disturbance vanishing in the far field. By assuming that $u \equiv 1$, applying the divergence operator to (44)-(46), and using (43), we see that the pressure in the far field behaves as $K_{n}(\alpha r)$, where $K_{n}$ is the modified Bessel function of order $n$. The behavior of the velocity components is then obtained by assuming a balance between the convective and pressure terms in (44)-(46). We can then use Neumann conditions of the type (36) for the pressure and the velocity components provided $r_{\max }$ is such that $\alpha r_{\max }$ is greater than 1. Two different ways of normalizing the problem were used; setting the streamwise component of the wall shear to one and setting the far field value of the pressure to a small arbitrary constant. In test cases both produced the same results. The former was used for the results presented below.

Again a standard finite difference method with a Newton iteration scheme was used. An axisymmetric version of the solver was produced and the results compared with those generated using the stream function $\phi$ as the dependent variable. There was no significant difference between the results. In particular, both methods produced the same critical Reynolds number. Also, for large Re, near the upstream end of the cylinder, at leading order the stability equations will reduce to those for the analogous three-dimensional problem in Cartesian coordinates [cf. (38)], and hence Squires transformation $^{6}$ should apply. Solutions were generated for $\operatorname{Re}=10^{5}$ and $n=5$. The results were compared with those found with $n=0$ with the lower Reynolds number obtained from Squires transformation, which for viscous problems, gives a change in the Reynolds number as well as the relevant wave number when mapping a three-dimensional disturbance to a two-dimensional one with the same value of $c$. Note, however, that in this case when the appropriate boundary layer scalings are adopted, i.e., $\beta=\alpha \operatorname{Re}^{-1 / 2}$ for the wave number and $\mathrm{Re}^{1 / 2}$ for the Reynolds number, as in (38), Squires transformation implies that the basic wave number $\alpha$ is the same for the two- and three-dimensional disturbances while $R_{2}=\left[\alpha^{2} /\left(\alpha^{2}+n^{2}\right)\right] R_{3}$ where $R_{2}$ and $R_{3}$ are the twoand three-dimensional Reynolds numbers, respectively. The level of agreement between the numerical and predicted results for the two-dimensional disturbance was as expected, i.e., consistent with the difference found above between the results from the axisymmetric calculations and the predictions from Blasius flow.

Table I gives critical Reynolds $\left(R_{c}\right)$ numbers for $n=1$ to $5, n=10$ and the axisymmetric mode $(n=0)$ and, for each $n$, a number of values obtained at the critical point $\left(x_{c}\right)$ where the flow first becomes unstable. Unlike for problems in Cartesian coordinates, the two-dimensional disturbance is not the least stable in the sense of having the lowest critical Reynolds number; it is only the fourth least stable mode with $n=1,2$, and 3 all having lower critical values.

The value of $x_{c}$ varies inversely with $R_{c}$ moving upstream as $R_{c}$ increases. In contrast, apart from the axisymmetric mode, the value of $\alpha$ at the critical points varies directly with $R_{c}$ and inversely with $x_{c}$. Even when including the basic $\mathrm{Re}^{-1 / 2}$ boundary layer scaling, producing $\beta_{c}$ from
TABLE I. Critical Reynolds numbers for different modes. $n=0$ is the axisymmetric disturbance. $c_{r}$ and $x_{c}$ give the approximate value of the wave speed and position of the neutral disturbance at the critical Reynolds number, $R_{b l}$ is the Reynolds number based on the boundary layer thickness at this point, $R_{x}=R_{c} x_{c}$ is the Reynolds based on the distance from the leading edge, and $\beta_{c}=\alpha_{c} R_{c}^{-1 / 2}$.

\begin{tabular}{lrcclccc}
\hline \hline$n$ & \multicolumn{1}{c}{$R_{c}$} & $x_{c}$ & $\alpha_{c}$ & \multicolumn{1}{c}{$\beta_{c}$} & $c_{r}$ & $R_{b l}$ & $R_{x}$ \\
\hline 0 & 12439 & 47.0 & 2.73 & 0.0245 & 0.317 & 3591 & $585 \times 10^{3}$ \\
1 & 1060 & 543 & 0.125 & 0.00385 & 0.552 & 3008 & $576 \times 10^{3}$ \\
2 & 6070 & 91.1 & 0.775 & 0.00995 & 0.422 & 3383 & $553 \times 10^{3}$ \\
3 & 10102 & 43.4 & 1.60 & 0.0159 & 0.403 & 3101 & $439 \times 10^{3}$ \\
4 & 13735 & 26.8 & 2.54 & 0.0217 & 0.398 & 2885 & $369 \times 10^{3}$ \\
5 & 17199 & 19.0 & 3.53 & 0.0269 & 0.396 & 2733 & $326 \times 10^{3}$ \\
10 & 33855 & 7.4 & 8.58 & 0.0466 & 0.396 & 2429 & $251 \times 10^{3}$ \\
\hline \hline
\end{tabular}

$\alpha_{c}$, this pattern of variation in the wave number holds. This reflects the change in the boundary layer thickness with distance downstream, and hence the implicit change in the scaling for the stability problem.

Apart from the axisymmetric mode, the wave speed at the critical point (Table I) decreases with $n$, and appears to be tending to a constant value as $n$ increases. This value is essentially the same as that given in Ref. 6 for Blasius flow. This is as expected since as Re increases the flow and its stability characteristics should approach those for Blasius flow. Hence from Squires transformation the value of $c_{r}$ at the critical point should be the same for all modes. Table I also gives Reynolds numbers $\left(R_{b l}\right)$ calculated using the boundary layer thickness at the critical point as the characteristic length and Reynolds numbers based on the distance from the leading edge $\left(R_{x}=R_{c} x_{c}\right)$. The values of $R_{b l}$ are more consistent than $R_{c}$, and for higher $n$ decrease rather than increase with $n$. Note that all the $R_{b l}$ in Table I are larger than the corresponding flat plate value of 1481. The values of $R_{x}$ at the critical point decrease with $n$, but all those given in Table I are well above the value for Blasius flow of $91 \times 10^{3}$. Also, although the values of $R_{c}$ for $n=0$ and $n$ $=1$ are an order of magnitude different, the values of $R_{x}$ at the critical point for these two modes are similar due to an almost equivalent but opposing change in the value of $x_{c}$.

For any particular value of $n \geqslant 1$, for Reynolds numbers above its critical value, the mode shows similar behavior with $x$ to that for the axisymmetric mode. That is, it is unstable for only a finite distance along the cylinder, with the flow returning to stability further downstream.

Figure 9 shows the maximum value of $c_{i}$ versus $x$ for the unstable modes for $\operatorname{Re}=15000$, with $x$ on a $\log$ scale for clarity. For the three-dimensional modes there is a common pattern; as $n$ and the critical Reynolds number increases, the peak value of $c_{i}$ decreases as does the size of the region in which the flow is unstable. The behavior of the axisymmetric mode is different; although $R_{c}$ is lower for $n=0$ than $n$ $=3, n=0$ has the larger region of instability. Also, for $n$ $=0, c_{i}$ has, in as far as is shown in Fig. 9, stopped decaying.

The wave speed $c_{r}$ corresponding to the values of $c_{i}$ shown in Fig. 9 is plotted against $x$ in Fig. 10. Again, the pattern for the three-dimensional modes is different than that for the axisymmetric mode. For $n>0$ there is a clear mini- 


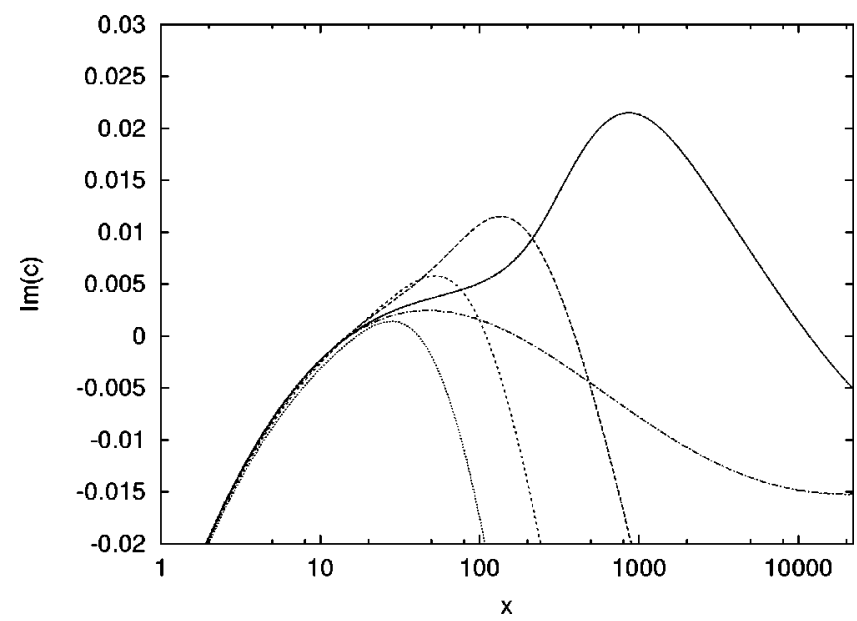

FIG. 9. The maximum value of $c_{i}$ versus $x$ for $\mathrm{Re}=15000$. In descending order of their maximum value the curves are for $n=1,2,3,0$, and 4 .

mum in the value of $c_{r}$, with the minimum value increasing and moving upstream as $n$ increases. In contrast, the wave speed for $n=0$ decreases monotonically over the region shown. For $n=1,2$, and 3 the minimum in $c_{r}$ occurs in the unstable region, while for $n=4$ it is very close to the upstream neutral point.

The wave number $\alpha$ corresponding to the values shown in Figs. 9 and 10 is plotted against $x$ in Fig. 11. In all cases $\alpha$ decreases with $x$. However, rather than tending to zero, which appears to be happening for $n>0$ in the region shown, for $n=0$, as with $c_{i}$ (Fig. 10), $\alpha$ appears to have leveled out.

Above, the maximum value of $c_{i}$ has been plotted against $x$ in Fig. 9. However, since the wave number also varies with $x$ (Fig. 11), the maximum value of $c_{i}$ may not correspond to the fastest growing mode. Further, the fact that easily the largest values of $c_{i}$ are for $n=1$ does not necessarily imply that $n=1$ is the most dangerous mode as its peak values of $c_{i}$ are downstream where $\alpha$ is small. A set of calculations was performed for $\mathrm{Re}=15000$ tracking the maximum growth rate rather the maximum value of $c_{i}$. The

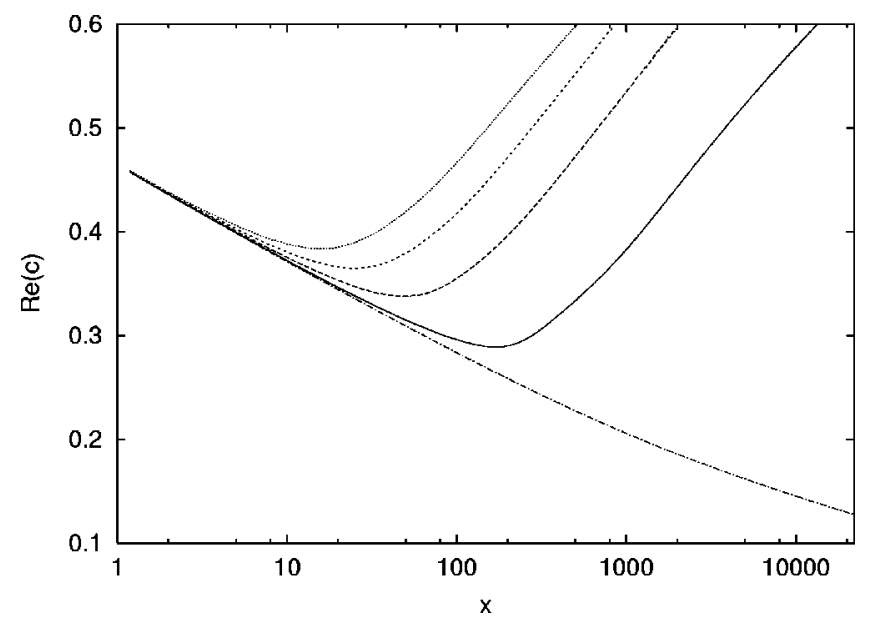

FIG. 10. The wave speed $c_{r}$ corresponding to the maximum value of $c_{i}$ versus $x$ for $\operatorname{Re}=15000$. From the bottom the curves are for $n=0,1,2,3$, and 4 .

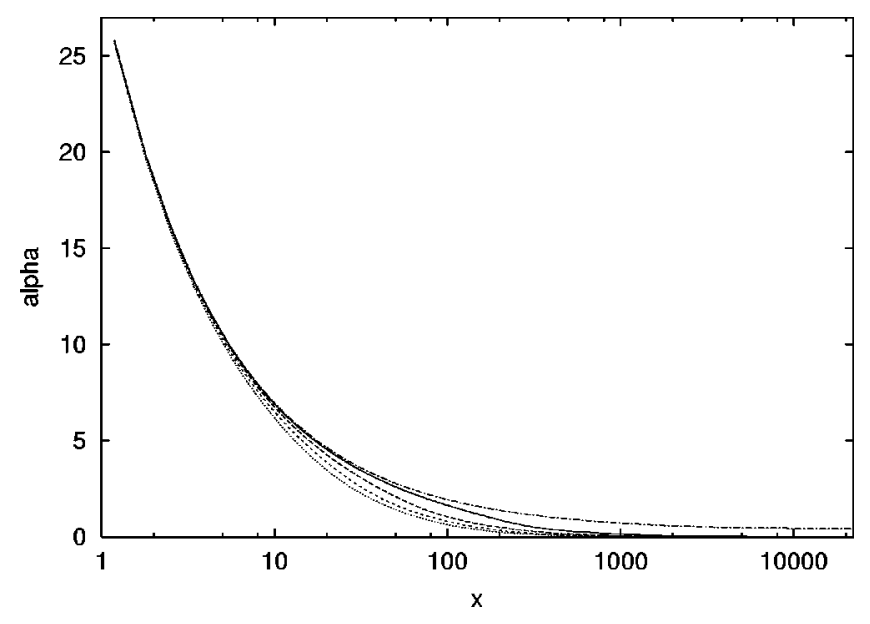

FIG. 11. The wave number $\alpha$ corresponding to the maximum value of $c_{i}$ versus $x$ for $\operatorname{Re}=15000$. From the top the curves are for $n=0,1,2,3$, and 4 .

results are shown in Fig. 12. From this figure it is seen that for $n=1$, the maximum value of $c_{i}$ does not give the most unstable mode over most of the region in which this mode is unstable. Also, far downstream, the growth rate appears to be tending to zero in Fig. 12 because of the very small values of $\alpha$ in this region. For the other modes, as $n$ increases, the difference between the maximum growth rate and that found with the maximum value of $c_{i}$ decreases. On the scale shown in Fig. 12 there is no difference between the two sets of growth rates for $n=0$ and 4 . Figure 12 also shows that for this Reynolds number, the least stable mode, in terms of the growth rate, is $n=2$, with $n=0$ only the fourth least stable mode, behind $n=2,3$ and 1 .

The neutral stability curves for $\operatorname{Re}=15000$ are shown in Fig. 13. For clarity, only the upstream portion of the curve is shown for $n=1$, although this curve does close far downstream at $x \approx 1.15 \times 10^{4}$, as indicated in Fig. 9. As can be

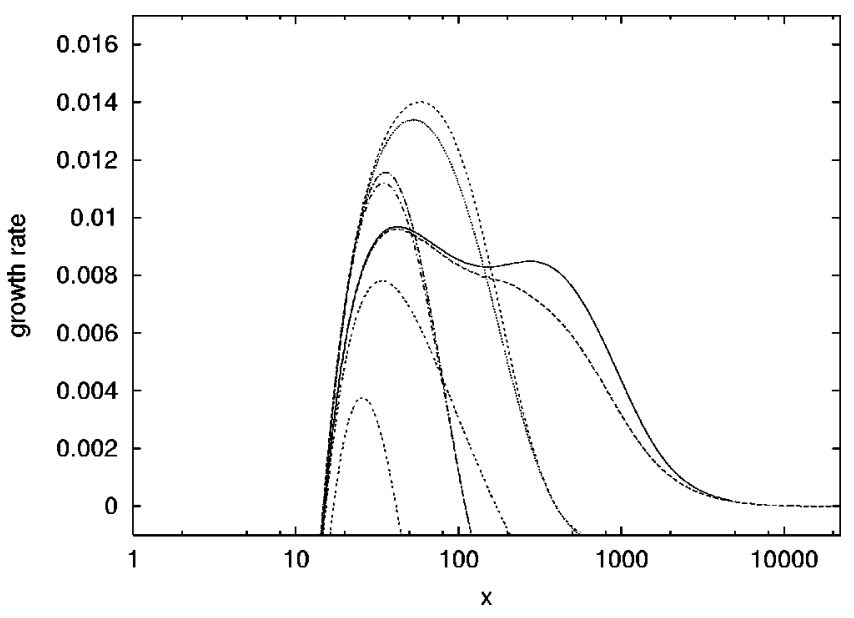

FIG. 12. Maximum growth rate $\alpha c_{i}$ versus $x$ for $\mathrm{Re}=15000$. The two curves extending downstream are for $n=1$, with the top one giving the maximum growth rate and the bottom the growth rate corresponding to the maximum value of $c_{i}$. The other curves, in terms of descending peak values, are for $n=2$ (maximum growth rate), $n=2$ (maximum value of $c_{i}$ ), $n=3$ (maximum growth rate), $n=3$ (maximum value of $c_{i}$ ), and $n=0$ and 4 . 


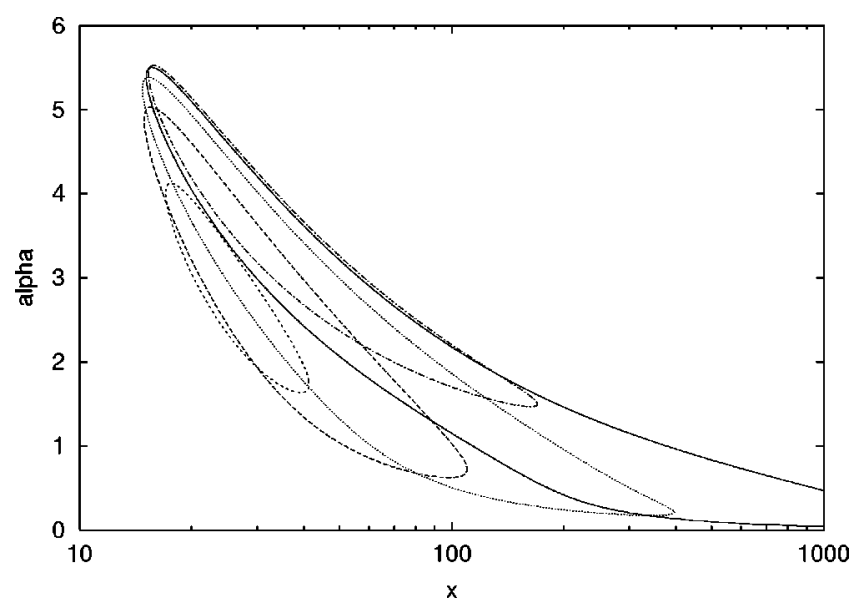

FIG. 13. Neutral curves for $\mathrm{Re}=15000$. The solid curve which is open at the right is for $n=1$. The other curves in terms of their maximum downstream extent are for $n=2,0,3$ and 4 .

seen, there are significant differences not only in the regions in which the various modes are unstable, but also in the wave numbers. For example, for $n=4$ there is no overlap with $n$ $=0$ or 1 in the range of unstable wave numbers. Also, it is clear that in the upstream region $n=2$ has the largest range of unstable wave numbers, which supports the idea that this would be the most dangerous mode for this Reynolds number.

As the Reynolds number increases the three dimensional modes show a similar pattern to that found for the axisymmetric modes, with the region of instability increasing but still finite. For example, for $\operatorname{Re}=10^{5}$ the $n=1$ mode is unstable from $x_{c} \approx 0.99$ to $x_{s} \approx 7.7 \times 10^{4}$.

\section{DISCUSSION}

Calculations have been performed for the boundary layer on a long thin cylinder, including effects which come from the radial nature of the problem. Near the leading edge of the cylinder a solution for the flow can be written as a series, as in (9)-(17). This series has Blasius flow as its leading term, and is formally valid for $x \ll \operatorname{Re}$. However, in practice the second term in the series, which relative to the leading term is of order $(x / \operatorname{Re})^{1 / 2}$, plays a significant role much closer to the leading edge. In the expression for the skin friction (18), the ratio of the second term to the leading term is $2.09(x / \mathrm{Re})^{1 / 2}$, which equals 0.66 when $x / \operatorname{Re}=1 / 10$, and 0.21 when $x / \operatorname{Re}=1 / 100$. Hence, except very close to the leading edge, $\tau$ is significantly different from that for Blasius flow. This can be seen clearly in Fig. 1 which shows the skin friction near the leading edge for $\operatorname{Re}=10^{4}$.

Further down the cylinder, when $x \gg \mathrm{Re}$, the series solution given by Glauert and Lighthill ${ }^{4}$ gives excellent results for the skin friction (Fig. 2), but worse agreement for the displacement area (Fig. 3).

The boundary layer thickness is lower and the skin friction is higher in the cylinder flow than those for Blasius flow. In general this would suggest that the flow is more stable. This prediction has been borne out. In fact, for flow with $\operatorname{Re}<1060$ or less the flow is unconditionally stable to linear normal mode disturbances. Further, unlike flows in Cartesian coordinates, two-dimensional disturbances are not the least stable in terms of having the lowest critical Reynolds number, with the $n=1$ mode having a critical Reynolds number an order of magnitude less than the axisymmetric $(n=0)$ mode.

The standard physical explanation for the existence of unstable normal modes in viscous flows that do not have an inflexion point in their velocity profile is that a phase difference in the transverse direction between the velocity components gives rise to a Reynolds stress which is sufficiently large to overcome the stabilizing dissipative effects of the viscosity (see, e.g., Ref. 7, p. 230). The equation governing the rate of change of the kinetic energy of the perturbations from a two-dimensional disturbance to a two-dimensional flow (Refs. 6 and 7) is

$$
\frac{d K}{d t}=\int_{0}^{\infty} u^{\prime}(y) \tau_{x y} d y-\frac{1}{\operatorname{Re}} \int_{0}^{\infty} \omega^{2} d y,
$$

where $K$ is the total kinetic energy of the disturbance, $\tau_{x y}$ is the Reynolds stress, and $\omega$ is the vorticity of the disturbance. All of these quantities have been averaged over a wavelength. Writing the perturbation velocity components as in (42) with $n=0$, the Reynolds stress is given by

$$
\tau_{x y}=-\overline{\hat{u} \hat{v}}=-1 / 2|\hat{u}(y)||\hat{v}(y)| \cos \left[\theta_{u}(y)-\theta_{v}(y)\right] e^{2 c_{i} t},
$$

where the overline denotes the averaging in $x$ and

$$
\hat{u}(y)=|\hat{u}(y)| e^{i \theta_{u}(y)}, \quad \hat{v}(y)=|\hat{v}(y)| e^{i \theta_{v}(y)} .
$$

In (47),

$$
\omega^{2}=1 / 2|\hat{\omega}(y)|^{2} \quad \text { where } \hat{\omega}(y)=i \alpha \hat{v}-\frac{\partial \hat{u}}{\partial y} .
$$

For the axisymmetric case the energy equation is

$$
\frac{d K}{d t}=\int_{1}^{\infty} u^{\prime}(r) \tau_{x r} r d r-\frac{1}{\operatorname{Re}} \int_{1}^{\infty} \omega^{2} r d r
$$

with $y$ in (48)-(50) replaced by $r$.

If $\theta_{u}(y)-\theta_{v}(y) \neq \pm \pi / 2$ then it is possible that the production term in (47) or (51) (the first term on the right-hand side) may outweigh the dissipation ( $\epsilon$, the second term on the right) and lead to growth in the energy of the disturbance. The growth rate of the kinetic energy normalized by the dissipation $(d K / d t /|\epsilon|)$ is shown in Fig. 14 for both the twodimensional and axisymmetric cases with $\operatorname{Re}=15000$. For the axisymmetric problem $d K / d t$ is positive only for a small region matching that with $c_{i}>0$ as shown in Figs. 9 and 12 . In contrast, for Blasius flow the normalized kinetic energy growth rate increases monotonically over the region shown. Examination of the two terms that make up $d K / d t$ shows that there is relatively little change in the dissipation, and that the difference in behavior is almost entirely due to the change in the rate of production of kinetic energy, i.e., in the first term on the right-hand side of (47) and (51). Comparing the two cases at a point where both are unstable (e.g., at $x$ $=50$ ) shows that the main reason for the difference in the production term is that, for disturbances of the same size, the 


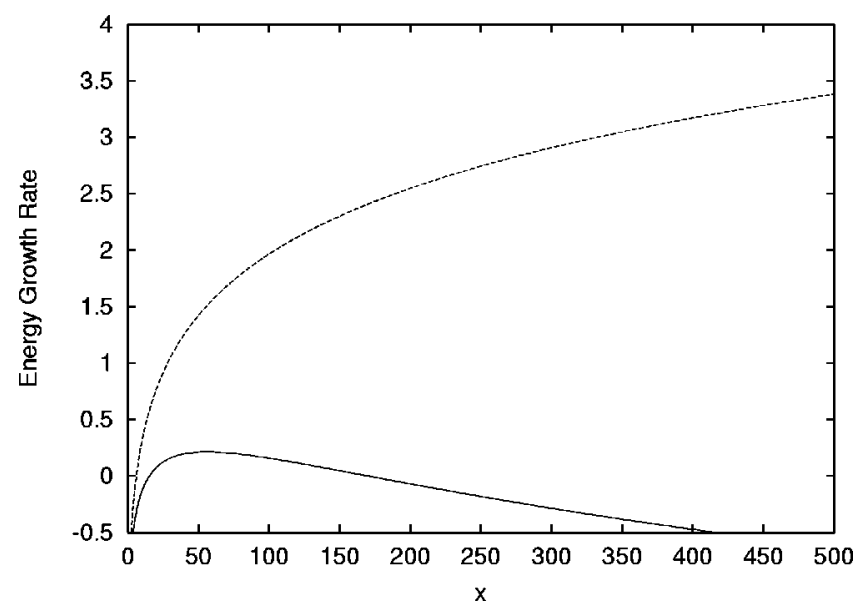

FIG. 14. Disturbance energy growth rate normalized by the dissipation $d K / d t /|\epsilon|$. The lower curve is for the axisymmetric case and the upper for Blasius flow.

Reynolds stress is significantly lower in the axisymmetric case than the two-dimensional flat plate case. That is, the primary reason for the enhanced stability characteristics in the axisymmetric case is that the disturbances are such that they generate lower levels of Reynolds stress, with a (relative) peak production rate near the leading edge, decreasing further downstream as the flow deviates further from Blasius flow. It follows that the growth rate of the total kinetic energy of the disturbance is lower than that for Blasius flow, and positive for only a finite length of the cylinder.

Both the change in shape of the velocity profile and the change in the governing equation for the disturbance play a role in generating the change in the disturbance and the stability of the flow. For $\mathrm{Re}=15000$ using either the axisymmetric stability solver with the Blasius profile or the twodimensional Orr-Sommerfeld solver with the axisymmetric flow profile gives stability results which lie between those for the two cases. Further, for $\mathrm{Re}=10^{4}$, both the OrrSommerfeld solver with the cylindrical velocity and the Blasius profile with the axisymmetric stability solver give unstable modes, although unconditional stability has been found for $n=0$ at this Reynolds number.
Physically the flow would still be expected to become unstable then turbulent along the cylinder as the boundary layer grows in thickness. However, at Reynolds numbers of $O\left(10^{4}\right)$ or less there will be no simple, two-dimensional, Tollmein-Schlichting-type wave growth/transition scenario, and for Reynolds numbers between $O\left(10^{3}\right)$ and $O\left(10^{4}\right)$ any linear, normal mode growth of the type considered here is necessarily three dimensional. Further, instability when it occurs is further downstream than for Blasius flow, and for the Reynolds numbers investigated in detail, occurs only for a finite length of the cylinder, with the flow becoming stable again when $x$ is still well short of Re. This does not of course imply that the flow will be laminar far downstream. However, with a carefully designed experiment it may be that laminar flow can be maintained much further downstream than with a flat plate (cf. Poiseuille flow in a pipe as opposed to a channel).

Unconditional normal mode linear stability has of course been found with other basic flows. In particular, no unstable modes have been found for Poiseuille flow in a pipe, although this flow is well known to be unstable at sufficiently high Reynolds number.

\section{ACKNOWLEDGMENT}

This work was supported by the Defense and Evaluation Research Agency (now QinetiQ Ltd.) as part of the MOD Applied Research Programmme.

${ }^{1}$ R. A. Seban and R. Bond, "Skin-friction and heat-transfer characteristics of a laminar boundary layer on a cylinder in axial compressible flow," J. Aerosp. Sci. 18, 671 (1951).

${ }^{2}$ H. R. Kelly, "A note on the laminar boundary layer on a cylinder in axial compressible flow," J. Aerosp. Sci. 21, 634 (1954).

${ }^{3} \mathrm{~K}$. Stewartson, "The asymptotic boundary layer on a circular cylinder in axial incompressible flow," Q. Appl. Math. 13, 113 (1955).

${ }^{4}$ M. B. Glauert and M. J. Lighthill, "The axisymmetric boundary layer on a long thin cylinder," Proc. R. Soc. London, Ser. A 224, 188 (1955).

${ }^{5}$ P. W. Duck, "Viscous flow through unsteady symmetric channels," J. Fluid Mech. 95, 635 (1979).

${ }^{6}$ P. G. Drazin and W. H. Reid, Hydrodynamic Stability Theory (Cambridge University Press, Cambridge, MA, 1981)

${ }^{7} \mathrm{P}$. Huerre and M. Rossi, in Hydrodynamics and Nonlinear Instabilities, edited by C. Godrecche and P. Manneville (Cambridge University Press, Cambridge, MA, 1998). 